\title{
Dural Arteriovenous Fistula Presenting with Exophthalmos, Hemiparesis and Seizures: Resolution after Endovascular Treatment - Case Report
}

\section{Aminur Rahman ${ }^{1 *}$, Sirintara Pongpech ${ }^{2}$, Pakorn Jiarakongmun², Ekachat Chanthanaphak ${ }^{2}$, Wittawat Takong ${ }^{2}$, Kittiphop Somboonnithiphol ${ }^{2}$, Thanaboon Worakijthamrongchai ${ }^{2}$ and Firoz Ahmed Quraishi ${ }^{3}$}

${ }^{1}$ Department of Neurology, Sir Salimullah Medical College, Bangladesh

${ }^{2}$ The Division of Interventional Neuroradiology, Department of Radiology, Ramathibodi Hospital, Faculty of Medicine, Mahidol University, Thailand

${ }^{3}$ Department of Neurology, Anwar Khan Modern Medical College, Bangladesh

*Corresponding Author: Aminur Rahman, Department of Neurology, Sir

Salimullah Medical College, Bangladesh.
Received: July 12, 2020

Published: August 26, 2020

(C) All rights are reserved by Aminur

Rahman, et al.

\section{Abstract}

A concomitant focal seizure with left hemipaeresis and exophthalmos in the context of an aggressive cavernous dural arteriovenous fistula (dAVF) is a variable neurological presentation. Here, we report a 73 years old female who presented with a 5-months history of progressive painless exophthalmos with conjunctival chemosis of right eye without any visual impairment. But now she had admitted to the hospital due to new onset of focal seizures with mild left hemipaeresis. Emergency MDCT scan brain showed suspected small malignant dural ateriovenous fistula of right sphenoparietal sinus with cerebral venous congestion and tiny hemorrhagic venous infarction at right operculum and right exophthalmos. Cerebral angiography demonstrated right cavernous dAVF fed by branches from the right ascending pharyngeal artery and right middle meningeal artery, right accessory meningeal artery, bilateral arteries of foramen rotendum, dural branch of left vertebral artery with drainage to the right superior ophthalmic vein, right sphenoparietal sinus and right frontal cortical vein which was consistent with Cognard Type IIb dAVF. Successful transvenous embolization of the right cavernous via the right inferior petrosal sinus was done by using fibre coils and NBCA. Post- embolization control angiogram showed complete obliteration of right cavernous dAVF feeding vessels. Subsequent clinical follow up revealed that she had dramatic improvement of proptosis, chemosis and neurological symptoms along with cessation of seizures.

Keywords: Dural Arteriovenous Fistula; Exophthalmos; Hemipaeresis; Seizures; Endovascular Treatment

\section{Abbreviations}

dAVF:DuralArteriovenous Fistula; OPD: Outpaient Department; NBCA: N-butyl-2-cyanoacrylate

\section{Introduction}

Intracranial dural arteriovenous fistulas (dAVF) are pathologic shunt situated between dural arteries and dural venous sinuses, meningeal veins or an adjacent cortical vein which can have profound detrimental effects on the underlying brain and cranial nerves [1]. It may also present in the spinal compartments of the central nervous system [2]. In intracranial, most frequently dAVF are affecting the region of the transverse and cavernous sinuses but uncommonly involve the dura of the craniocervical junction [3]. Intracranial dAVF account for $10-15 \%$ of all intracranial artriovenous lesions [4]. The incidence of $\mathrm{dAVF}$ at various locations as reported in literature is as follows: transverse sinus $50 \%$, cavernous sinus $16 \%$, tentorium cerebelli $12 \%$, and superior sagittal sinus, $8 \%$ [5]. Dural AVF can affect a variety of cerebral venous structures, which can present at various clinical stages, ranging from a simple irritat- 
ing pulse-synchronous bruit, tinnitus to a disabling neurological deficit as a result of ischaemia from impaired venous drainage to life threatening intracranial hemorrhage from venous hypertension $[5,6]$. In our case report on emphasize on the neurological presentations in intracranial dAVF along with treatment.

\section{Case Report}

A 73 years old hypertensive and diabetic female was attended to the outpatient department (OPD) with the history of left hemipaeresis with focal seizure on the left upper limb without generalization for 1 week. But initially she noticed that her right eye was congested and gradually painless proptosed without any visual impairment. She had no complaints of tremulous hand, weight loss, palpitation, thyromegaly, fever or head trauma for last 5 months. Despite of initial symptoms she was not consulted to any physicians or even the ophthalmologist within that time. After appearing of neurological features she was admitted into a tertiary level hospital and from their sent to our institute. Her general physical examination was revealed normal and neurological examination was found well orientated, upper motor neuron lesion in right side (MRC Grade 4) without any cranial nerve involvement. Laboratory investigations including thyroid, renal and liver function tests were normal. Emergency MDCT scan brain showed suspected small aggressive dAVF of right sphenoparietal sinus with cerebral venous congestion and tiny hemorrhagic venous infarction at right operculum and right exophthalmos (Figure 1). With above clinical findings along with neurological involvements and suspicious aggressive dAVF lesion in MDCT so we went for specific cerebral angiography inspite of Magnetic resonance imaging (MRI) and MR angiography (MRA).
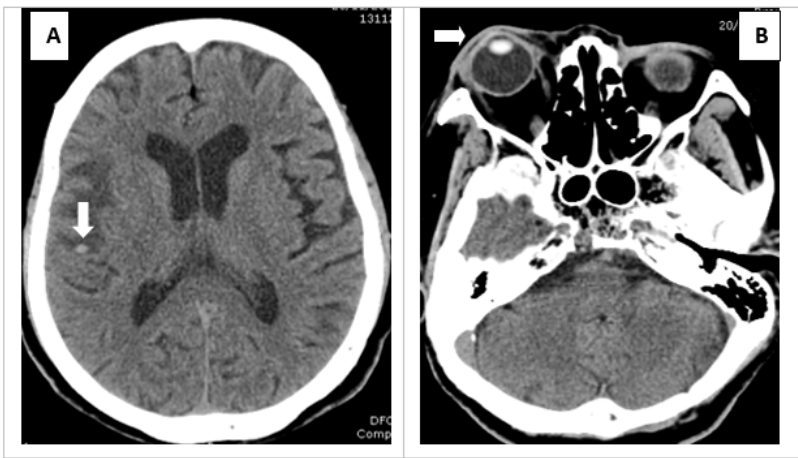

Figure 1: MDCT showed suspected small aggressive dAVF of right sphenoparietal sinus with cerebral venous congestion and tiny hemorrhagic venous infarction at right operculum (Figure: A-arrow head) and right proptosis (Figure: B -arrow head).
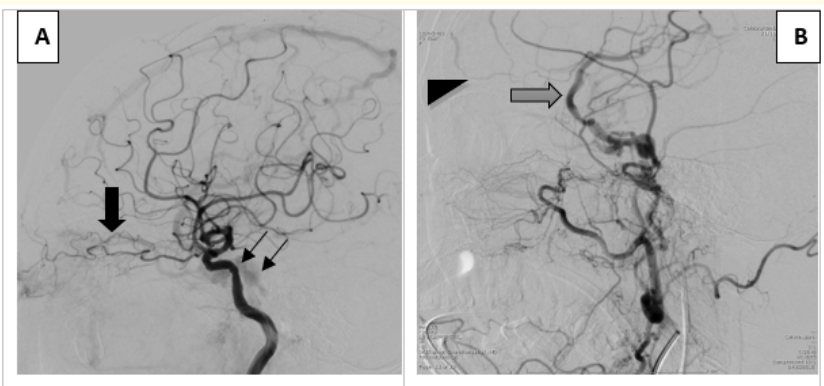

Figure 2: $(A+B)$ Cerebral angiography revealed right cavernous $\mathrm{dAVF}$ (double arrow) with drainage to the right superior ophthalmic vein (bold black arrow), right sphenoparietal sinus (bold white arrow) and right frontal cortical vein (black arrow head).
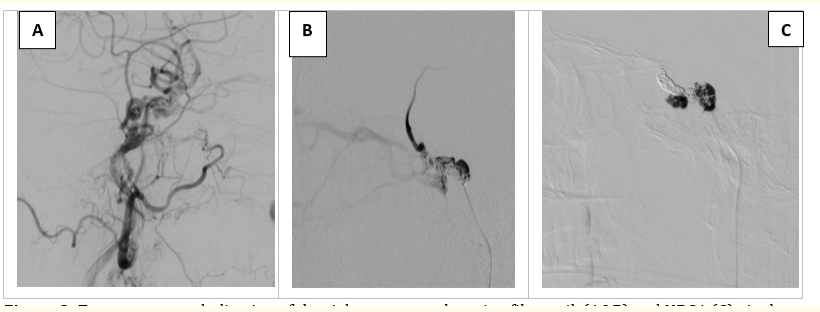

Figure 3: Transvenous embolization of the right cavernous by using fibre coils(A\&B) and NBCA (C) via the right inferior petrosal sinus.

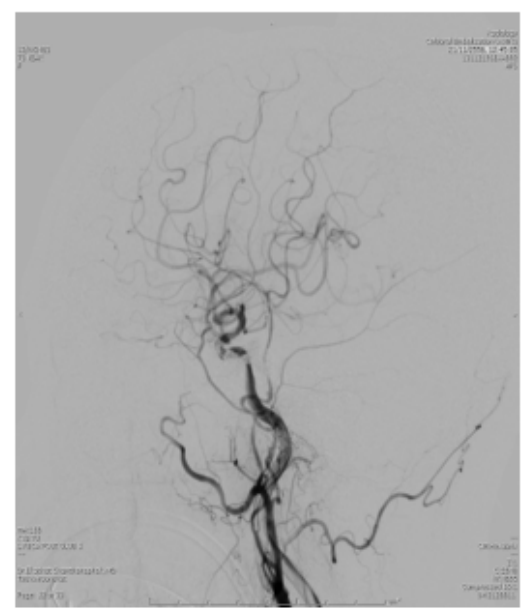

Figure 4: Post-embolization control angiogram shows complete obliteration of right cavernous dAVF feeding vessels. 

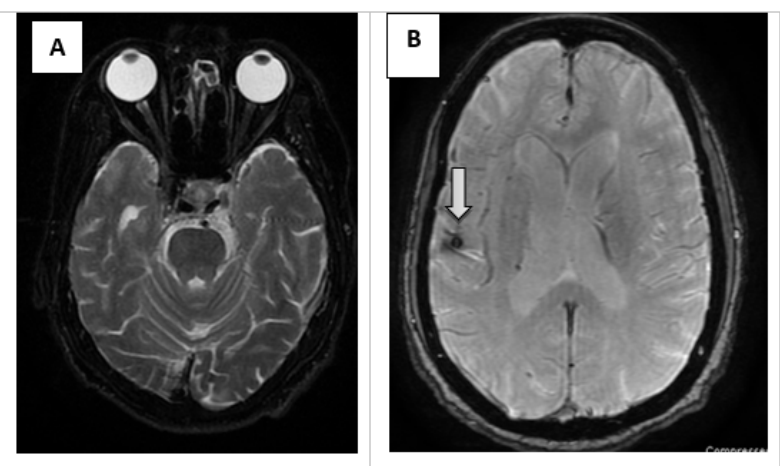

Figure 5: Follow up MRI and MRA after 3 months of post embolization found tranavenous embolization coils and glue without residual $\mathrm{dAVF}$ with resolution of venous congestion at anterior right temporal lobe and right frontal operculum without proptosis (A), leaving only a few tiny hemorrhagic spots and superficial siderosis (arrow head-B).

Cerebral angiography revealed right cavernous dAVF which was fed by branches from the right ascending pharyngeal artery and right middle meningeal artery, right accessory meningeal artery, bilateral arteries of foramen rotendum, dural branch of left vertebral artery with drainage to the right superior ophthalmic vein, right sphenoparietal sinus and right frontal cortical vein (Figure 2) which was consistent with Cognard Type IIbdAVF.

So we planned for embolization immediately as this was aggressive dAVF. Successful transvenous embolization of the right cavernous via the right inferior petrosal sinus was done by using fibre coils(Nester) initially but there was no complete obliteration of dAVF (Figure $3 \mathrm{~A}$ and $3 \mathrm{~B}$ ). Then we further embolized by $30 \%$ NBCA mixing with lipiodol (Figure $3 \mathrm{C}$ ) and post-embolization control angiogram showed complete obliteration of right cavernous dAVF feeding vessels (Figure 4). Her postoperative course was uneventful and neurologic deficits gradually improved. She underwent aggressive rehabilitation for 0.232 weeks after embolization and was conversant and ambulatory with minimal assistance at discharge with significant improvement of neurological symptoms. She had advised to attend the clinic for follow-up examinations 1 month, 3 months, and 5 months after endovascular treatment. At her 3-months follow-up we had done MRI and MRA and found tranavenous embolization coils and glue and no evidence of aforementioned residual dAVF with resolution of venous congestion at anterior right temporal lobe and right frontal operculum without proptosis (Figure 5A), leaving only a few tiny hemorrhagic spots and superficial siderosis (arrow head -Figure 5B). Subsequent clinical follow up revealed that she had dramatic improvement of proptosis, chemosis and neurological symptoms along with cessation of seizures.

\section{Discussion}

Approximately 10 to $15 \%$ of all intracranial arteriovenous malformations consist of adult-type DAVFs [7]. There is no correlation of dAVF between age, sex and frequency of aggressive neurologic symptoms $[8,9]$. The incidence of dAVFis a higher in women and mean age is between 40 and 60 years $[10,11]$ but dAVFs can occur at any age. In our reported study showed that the woman was 73years which was similar to the previous studies.

Although the mechanism of underlying the formation of fistulas is not yet fully understood but there are two hypotheses. Fistulas are thought to develop when normal physiologic pathways become pathologic arteriovenous shunts, or they are attributed to the release of other angiogenic factors that cause the shunts to develop [12]. The dAVFs are usually considered to be acquired lesions. Trauma is a well-known cause of acute onset dAVF attributed to damage of nearby dural arteries and veins. The DAVFs also have been reported by subacutely after trauma, infection and surgery. Thrombosis of a vein is also a common feature of dAVFs [13]. Our patient had no past history of trauma, infection, or surgery.

A wide spectrum of symptoms exists, ranging from the benign to the more aggressive; depend on the specific location of the lesion, extent of arterial supply and specific pattern of venous drainage. Drainage of a petrous region dAVF to the transverse or sigmoid sinus commonly produces pulsatile tinnitus; sometimes in association with an audible bruit [7]. Ocular manifestations (e.g.; opthalmoplagia, proptosis, chemosis, decreased visual acuity, bilateral papilloedema and atrophic optic disc) are commonly seen with carotid-cavernous dAVF $[13,15]$. Our reported case symptoms' began with ocular manifestations those were matched with the fistula in carotid-cavernous site.

More aggressive dAVF may manifest as focal neurological deficits, seizure, headache, progressive dementia-type of syndrome or cerebral haemorrhage, including subarachnoid, subdural or intraparenchymal bleeds and also brain edema and/or ischemia due to local venous congestion [13,16-18]. Such features are usually considered to be due to venous hypertension, although neurological deficits may be secondary to arterial steal [13]. In a meta-analysis of $360 \mathrm{dAVFs}$ the tentorial incisura was the most ominous location, with 31 out of 32 cases associated with haemorrhagic or non 
haemorrhagic stroke $[13,16]$. So the pattern of venous drainage was considered of paramount importance in predicting aggressive behavior. This case study had the manifestation of neurological symptoms due to long standing presentation as a result of venous hypertension.

CT, MRI and cerebral angiography all have roles to play in the investigation of patients with a possible DAVF. The differentiation of high- and low-risk DAVFs is based on venous drainage patterns: Increased cortical venous reflux is consistent with a more aggressive course $[10,19]$. Because the clinical and imaging features can be non-specific, the diagnosis of a DAVF is often delayed or missed, but for detection of these fistulas, cerebral angiography is the method of choice, allowing the dynamic assessment of cerebral circulation [20]. Benign diseases, without cortical venous reflux that can be missed using both CT and MRI, if there is a strong clinical suspicion of a fistula. If haemorrhage is suspected, nonenhanced CT is a pre-requisite. Venous congestion may appear as an area of low density on CT. Our studied case had showed in the Multi-detector CT (MDCT) with suspected small aggressive dAVF of right sphenoparietal sinus with cerebral venous congestion and tiny hemorrhagic venous infarction at right operculum and right exophthalmos.

In the most institutions CT is more readily available and cheaper than MRI and so becomes the first-line investigation of patients presenting with tinnitus, headache or other vague neurological symptoms. MDCT angiography (MDCTA) can now provide high resolution detail of vascular anatomy [9]. T2 weighted MRI is more sensitive to the white matter changes of venous congestion or infarction when compared to CT. It has the drawback of being less sensitive to the changes of acute haemorrhage. If dilated cortical veins are present they may be seen on conventional spin echo sequences and visualized using MR angiographic techniques such as phase contrast venography or contrast enhanced MR angiography $[9,21]$. Time-of flight MRA (TOF MRA) is now widely available test for primary screening tool for when DAVF is suspected [22]. However, studies have reported that CTA has reduced sensitivity versus MRA for the detection of dAVFs (15.4\% versus 50\%) [23]. Cerebral angiography is the gold standard for detection of cerebral dAVF [24]. So we studied cerebral angiography and found right cavernous dAVF which was fed by branches from the right ascending pharyngeal artery and right middle meningeal artery, right accessory meningeal artery, bilateral arteries of foramen rotendum, dural branch of left vertebral artery with drainage to the right superior ophthalmic vein, right sphenoparietal sinus and right frontal cortical vein which was consistent with Cognard Type IIb classification.
Treatment is dependent on the clinical picture, temporal progression and the grade of fistula [25]. High risk radiographic features, such as cortically diverted venous drainage, patient with visual loss, hemorrhage or infarction require prompt therapy. A multidisciplinary approach involving an interventional neurologist, neurosurgeon and neuroradiologist is required. The goal of treatment of high risk fistulas should be complete obliteration. Treatment modalities include conservative management, endovascular intervention (i.e. transvenous transarterial embolization), microsurgery, and stereotactic radiosurgery [17]. Although endovascular embolization has evolved to become the first line treatment option for most dAVFs, it can be limited by inadequate access to the fistula point, non-target embolization, and the potential for recanalization [26]. In these instances, definitive cure can be achieved by surgical interruption of leptomeningeal venous drainage [26].

The low risk fistulas can be treated conservatively. Transarterial embolization (TAE) of feeding artery through external carotid branches with particle can easily performed, which can reduce shunt flow. TAE with n-butyl-2-cyanoacrylate has been applied to complex dural AVFs that are not accessible with percutaneous transvenous catheterization. However, complete cures are difficult from this method because of the existence of feeding arteries that cannot be catheterized and the recruitment of a blood supply from collateral arteries [27]. Therefore, this method is generally used to relieve symptoms or in combination with other procedures such as transvenous embolization (TVE), irradiation or surgery $[7,28]$.

TVE with coils must be considered in patients with fistulas of the transverse and sigmoid sinuses. For curative purposes TVE with coils is used and many studies have reported it to be very useful (complete occlusion in $80 \%-100 \%$ of cases) $[7,29]$. But this procedure sometimes associated with, serious complications like; vessel injury and intracranial haemorrhage have also been reported [30]. Inadequate embolization leads to a worsening of symptoms. Critical assessment of diagnostic images and clinical conditions is also important for successful procedures. Previously this procedure was performed by direct surgical or transfemoral introduction of thrombogenic material into fistulae [31].

The use of detachable coils before liquid adhesives (onyx or glue) injection slows and decreases flow in the fistula and provides secure anchoring to the onyx or glue cast [32]. Other options such as surgical approaches and a combination of TAE and radiosurgery should also be considered when treating complex dAVFs [33]. Our case report demonstrated the aggressive dAVF, the presence of neu- 
rological features with cortical venous reflux. Therefore our goal of treatment was to complete obliteration of fistula. We planned initially transvenous coil embolization with fibre coils (nester) of the right cavernous sinus via the right inferior petrosal sinus. But after embolization of the right cavernous sinus there was still presence of reflux in the right sphenoparietal sinus. So we further embolized by 30\% NBCA mixing with lipiodol successfully and found complete obliteration dAVF in post-control angiography. Follow up MRI and MRA after 3 months of post embolization found tranavenous embolization coils and glue without residual dAVF with resolution of venous congestion at anterior right temporal lobe and right frontal operculum without proptosis (Figure 5A), leaving only a few tiny hemorrhagic spots and superficial siderosis (arrow head- Figure 5B). Subsequent clinical follow up revealed that she had dramatic improvement of proptosis, chemosis and neurological symptoms along with cessation of seizures.

\section{Conclusion}

Prognosis of high grade dAVFs greatly depends on early diagnosis and treatment. Significant advancements in the management of dAVFs have been made due to the development of recent pre- and intraoperative imaging methods with endovascular techniques. So the aggressive dAVFs need treatment immediately to prevent the further dreadful complications.

\section{Bibliography}

1. Kwon BJ., et al. "MR imaging findings of intracranial dural arteriovenous fistulas: relations with venous drainage patterns". AJNR American Journal of Neuroradiology 26 (2005): 2500-2507.

2. Robert WH., et al. "Dural arteriovenous fistulas of the craniocervical junction”. Skull Base Surgery 9.1 (1999).

3. Kirsch M., et al. "Endovascular management of dural arteriovenous fistulous of the transverse and sigmoid sinus in 150 patients". Neuroradiology 51 (2009): 477-483.

4. Newton TH and Cronqvist S. "Involvement of dural arteries in intracranial arteriovenous malformations". Radiology 93 (1969): 1071-1078.

5. Lasjaunias P., et al. "Neurological manifestations of intracranial duralarteriovenous malformations". Journal of Neuroradiology 64 (1986): 724-730.

6. Barnwell SL., et al. "A variant of arteriovenous fistulas within the wall of dural sinuses: Results of combined surgical and endovascular therapy". Journal of Neuroradiology 74 (1991): 199-204.
7. Vinuela F., et al. "Unusual clinical manifestations of duralarteriovenous malformations". Journal of Neuroradiology 64 (1986): 554-558.

8. Hirok K., et al. "Treatment of Intracranial Dural Arteriovenous Fistulas: Current Strategies Based on Location and Hemodynamics, and Alternative Techniques of Transcatheter Embolization". Radio Graphics 24 (2004): 1637-1653.

9. Stephan M., et al. "Arterialization of Cerebral Veins on Dynamic MDCT Angiography: A possible sign of a Dural Arteriovenous Fistula". AJR 184 (2005): 1313-1316.

10. Cognard C., et al. "Cerebral duralarteriovenous fistulas: Clinical and angiographic correlation with a revised classification of venous drainage”. Radiology 194 (1995): 671- 680

11. Davies MA., et al. "The validity of classification for the clinical presentation of intracranial duralarteriovenous fistulas". Journal of Neuroradiology 85 (1996): 830-837.

12. Hamada Y., et al. "Histopathological aspects of duralarteriovenous fistulas in the transverse-sigmoid sinus region in nine patients". Neurosurgery 40 (1997): 452-456.

13. Will A and Peter W. "Intracranial duralarteriovenous fistulae". Advances in Clinical Neuroscience and Rehabilitation 7.3 (2007): 10-12.

14. Yesim A., et al. "Intracranial Dural Arteriovenous Fistula Draining into Spinal Perimedullary Veins: A Rare Cause of Myelopathy". Journal of Korean Medical Science 21 (2006): 958-962.

15. Tsung J W., et al. "Ophthalmic Manifestations of Intracranial Dural Arteriovenous Fistula - Report of Four Cases". Tzu Chi Medical Journal 17.2 (2005): 93-97.

16. Awad IA., et al. "Intracranial dural arteriovenous malformations: factors predisposing to an aggressive neurological course". Journal of Neuroradiology 72 (1990): 839-850.

17. Cognard C., et al. "Dural arteriovenous fistulas as a cause of intracranial hypertension due to impairment of cranial venous outflow". Journal of Neurology, Neurosurgery, and Psychiatry 65 (1998): 308-316.

18. Hasumi T., et al. "Focal dural arteriovenous fistula (DAVF) presenting with progressive cognitive impairment including amnesia and alexia". Internal medicine 46.16 (2007): 1317-1320

19. Borden JA., et al. "A proposed classification for spinal and cranial duralarteriovenous fistulous malformations and impli- 
cations for treatment". Journal of Neuroradiology 82 (1995): 166-179.

20. Stephan M., et al. "Arterialization of Cerebral Veins on Dynamic MDCT Angiography: A Possible Sign of a Dural Arteriovenous Fistula". AJR American Journal of Roentgenology 184 (2005): 1313-1316.

21. Stephan G W., et al. "Cerebral Dural Arteriovenous Fistulas: Detection by Dynamic MR Projection Angiography". AJR American Journal of Roentgenology 174 (2000): 1293-1295.

22. Hirai T., et al. "Usefulness of source images from three dimensional time-of-flight MR angiography after treatment of cavernous duralarteriovenous fistulas". Radiation medicine 21.5 (2003): 205-209.

23. Cohen SD., et al. "Dural arteriovenous fistula: diagnosis, treatment, and outcomes”. Laryngoscope 119 (2009): 293-297.

24. Brown RD Jr., et al. "Natural history, evaluation, and management of intracranial vascular malformations". Mayo Clinic Proceedings 80 (2005): 269-281.

25. Ishii K., et al. "High risk Dural arteriovenous fistulae of transverse and sigmoid sinuses". American Journal of Neuroradiology 8 (1988): 1113-1121.

26. Gross BA and Du R. "Surgical treatment of high grade dural arteriovenous fistulae". Journal of Clinical Neuroscience 20 (2013): 1527-1532.

27. Kawaguchi S., et al. "Surgery for duralarteriovenous fistula in superior sagittal sinus and transverse sinus". Journal of Clinical Neuroscience 7 (2000): 47-49.

28. Jun S K., et al. "Transcranial Approach for Arterial Embolization of Dural Arteriovenous Fistula Within the Wall of the Superior Sagittal Sinus: A Case Report". Neurointervention 2 (2007): 117-121.

29. Roy D and Raymond J. "The role of transvenous embolization in the treatment of intracranial duralarteriovenous fistulas". Neurosurgery 40 (1997): 11331144.

30. Houser OW., et al. "Arteriovenous malformation affecting the transverse dural sinus-an acquiredlesion". Mayo Clinic Proceedings 54 (1979): 651-661.

31. Nelson PK., et al. "Use of a wedged microcatheter for curative transarterial embolization of complex intracranial dural arteriovenousfistulas: indications, endovascular technique, and outcome in 21 patients". Journal of Neuroradiology 98 (2003): 498-506.

32. Quinones D., et al. "Embolization of dural cavernous fistulas via superior ophthalmic vein approach". AJNR American Journal of Neuroradiology 18 (1997): 921-928.

33. Friedman JA., et al. "Results of combined stereotactic radiosurgery and transarterial embolization for dural arteriovenous fistulas of the transverse and sigmoid sinuses". Journal of Neuroradiology 94 (2001): 886-891.

\section{Assets from publication with us}

- Prompt Acknowledgement after receiving the article

- Thorough Double blinded peer review

- Rapid Publication

- Issue of Publication Certificate

- High visibility of your Published work

Website: www.actascientific.com/

Submit Article: www.actascientific.com/submission.php

Email us: editor@actascientific.com

Contact us: +919182824667 